\title{
Violencia de género en la televisión. Un análisis del discurso
}

\author{
Gender Violence on television: a discourse analysis \\ Vanesa Cruz-Olvera ${ }^{a}$, Gelacio Guzmán-Díaz ${ }^{b}$, Jesús Cisneros-Herrera ${ }^{c}$
}

\begin{abstract}
:
Gender violence, defined as any kind of violence towards women due to gender issues, is deeply rotted in societies. It is argued that this kind of violence is linked to socialization instruments, such as communication media. Approaching gender violence as an entertainment object where violence against women becomes a fetish hides the problem, promoting misunderstanding of this situations and of women who experience violence. In this paper, a non-probabilistic convenience sampling was used to select a tv show and a newscast to analyse the discourse about gender violence. It was found a tendency to hide violence towards women. These analysed tv broadcasts show the audience that the only choice for women to escape from violence is legal complaint. However, the tv broadcasts justify systematically the actions of aggressor and questioning the attacked women hiding any violence that have physical consequences.
\end{abstract}

Keywords:

Discourse analysis, gender violence, television

Resumen:

La violencia de género, definida como toda violencia ejercida hacia las mujeres por cuestiones de género, se encuentra fuertemente arraigada en las sociedades. Se alude que la perpetuación de este tipo de violencia está ligada a instrumentos de la socialización como lo son los medios de comunicación. El abordaje de la violencia de género como un objeto de entretenimiento en donde se fetichiza la violencia hacia la mujer se vuelve un factor que invisibiliza el problema, generando incomprensión hacia la situación y hacia las mujeres que sufren dicha violencia. En la presente investigación, se empleó un muestreo no probabilístico por conveniencia a partir del cual se eligieron un programa de espectáculos y uno de noticias para realizar el análisis del discurso de los mismos; se encontró una tendencia a invisibilizar la violencia a la mujer. Los programas analizados muestran a los televidentes que la única opción que tiene la mujer para salir de la violencia es la denuncia; sin embargo, resultan revictimizantes, se encuentran constanmente justificando las acciones del agresor y dudando de las mujeres agredidas invisibilizando toda violencia que no tenga consecuencias físicas.

Palabras Clave:

Análisis del discurso, televisión, violencia de género

\section{Introducción}

La violencia es un fenómeno que podría catalogarse como inherente a la humanidad, su presencia no puede establecerse en un momento preciso de la historia humana debido a su larga trayectoria; sin embargo, en la sociedad actual la violencia goza de no pocas penalizaciones buscando, sino su completa erradicación, una disminución de sus expresiones y alcances. Entre las diversas formas de violencia puede encontrarse la violencia de género a la que las Naciones Unidas (1993) define como como toda aquella violencia que se ejerce hacia las mujeres por cuestiones de género y que produce daños a nivel físico, psicológico o sexual en un ámbito público o privado.

La violencia está arraigada a las sociedades debido a la construcción de las mismas, Galarza, Cobo y Esquembre

\footnotetext{
Autor de Correspondencia, Universidad Autónoma del Estado de Hidalgo, https://orcid.org/0000-0002-6074-6336, Email: cr379301@uaeh.edu.mx

b Universidad Autónoma del Estado de Hidalgo, https://orcid.org/0000-0003-4869-8658, Email: gelacio_guzman@uaeh.edu.mx

c Universidad Autónoma del Estado de Hidalgo, https://orcid.org/0000-0003-1370-8086, Email: jesus_cisneros@uaeh.edu.mx
} 
exponen que la sociedad está compuesta por "estructuras materiales y simbólicas" (2016, p. 821) estas estructuras deben ser congruentes entre sí a fin de tener la fuerza necesaria para coaccionar a los actores sociales, es decir, las instituciones y las definiciones sociales son complementarias y de ellas se desprenden los valores dominantes de la sociedad los cuales se aceptan como circunstancias imposibles de cambiar gracias al proceso de socialización. Esto ocurre con la violencia de género en donde la víctima está en 'consenso' con su agresor (Galarza, Cobo y Esquembre, 2016).

Uno de los instrumentos que se utilizan para la socialización son los medios de comunicación gracias a los cuales la gente genera una opinión pública de los hechos en función a la forma en la que son presentados (Zurbano y Liberia, 2014). Para Galarza, Cobo y Esquembre (2016) los medios de comunicación responden a las instituciones sociales que siguen los preceptos del sistema patriarcal, por lo tanto, están destinados a invisibilizar la violencia ejercida por el hombre y perpetuar y aceptar la violencia simbólica y material hacia la mujer.

Núñez y Fernández (2015) aluden que los medios de comunicación son agentes que logran atraer la mirada del público a la violencia dándole visibilidad y reconocimiento, pese a ello, esta representación no incita a la "reflexión política y a la activación de la dimensión política” (Núñez y Fernández, 2015, p. 273) se construye una imagen de la víctima como sujeto pasivo, las mujeres son carentes de 'agencia' entendida como la capacidad que tienen las mujeres para lograr el empoderamiento a través de acciones (Butler, 1990 en Gámez y Núñez, 2013).

De esta manera se construye a la violencia de género como objeto de consumo en donde se eclipsan las formas de transformación a través de los discursos y lenguajes de acción social. A fin de crear una identidad colectiva para hacer inteligible la violencia hacia la mujer se hacen reiteraciones de la fetichización y objetualización de la misma; a la víctima se le subordina debido a la construcción mediática y a la posición de poder y prestigio que posee el individuo que presenta la noticia (Gámez,2012). Los medios de comunicación construyen una imagen de cómo debería verse una víctima y la sociedad invisibiliza a cualquiera que no tenga dichas características, los espectadores consideran que debe sentir compasión y un sentimiento de protección hacia aquella que no tiene voz, pero también se le responsabiliza (Gámez, 2012) se centran en el acto de violencia, en la relación que sostenía con el agresor, en sí tomó acciones legales (Gámez y Núñez, 2013)

Los noticieros se alejan de su función de educar y sensibilizar a los televidentes y se enfocan en objetualizar a la mujer, perpetuando el papel de víctima como un agente pasivo (Gámez, 2012), la violencia que más se presenta ante el público es aquella que tiene consecuencias físicas o visibles en la mujer, siendo la violencia simbólica o psicológica desprovista de la atención que merece (Zurbano y Liberia, 2014), se busca una visión dramática de los hechos que los televidentes puedan consumir y se eligen mujeres cuyas circunstancias atraigan, por ejemplo, si son indigentes o su nivel socioeconómico es bajo; sin embargo, también se refuerza que la violencia es un destino individual en donde la mujer que no se capaz de acatar las normas sociales está destinada a la tragedia.

Por su parte, en los programas de espectáculos también se abordan temas de violencia de género, pero esta exposición termina siendo un invisible social que como definen Giberti y Fernández (1989) son todos aquellos acontecimiento 0 hechos que al ser constantemente reproducidos es difícil visualizarlos pues se aíslan de toda comprensión.

Velázquez (2003) expone que todas estas situaciones de violencia al ser abordadas de esta manera, generan cierta fascinación hacia la violencia que se ejerce, la televisión mancilla la intimidad de víctima y la vuelve un objeto que puede consumirse, los espectadores ven esto como un hecho natural al eliminar la carga negativa implícita. En palabras de Zurbano y Liberia "los medios de comunicación simplifican la violencia de género transmitiéndola dentro de un marco de afectividad que, en clara conexión con las descripciones legales del problema a nivel nacional, obvian marcos interpretativos más amplios como los feministas" (2016, p. 125).

\section{Método}

La presente investigación se encuentra dentro de los parámetros de la metodología cualitativa, siendo además de alcance descriptivo.

Este estudio utilizará al análisis del discurso como método de obtención de información. De acuerdo a Sisto (2012), el lenguaje puede concebirse como el medio por el cuál las personas construyen sus realidades, modificable de acuerdo al contexto y a lo que se desee lograr con él, por ende, la psicología discursiva reconoce la incidencia de las variables sociales en la construcción de los individuos, siendo el habla y el discurso herramientas primordiales en esta construcción.

La realidad se representa a partir de un punto de particular del lenguaje y de la cultura, es por ello que es importante cuestionar lo que se presenta dentro de nuestro contexto (Magnabosco, 2014), para ello puede hacerse uso del construccionismo social que contempla que el conocimiento se adquiere en función de la cultura y de la interacción que sostienen las personas, esta visión posmoderna menciona que debe debatirse el 
conocimiento adquirido, no para conocer una verdad, sino para descubrir los significados que una persona encuentra de acuerdo a sus marcos culturales y crear significados compartidos (Magnabosco, 2014).

Dentro de este estudio se seleccionaron, a través de un muestreo probabilístico por conveniencia, programas vigentes de la televisión mexicana. Se eligieron dos tipos de programación: noticieros y programas de espectáculos. De cada programa se eligieron dos emisiones en las que se abordan noticias o situaciones de violencia de género, dando un total de 4 unidades de análisis.

\section{Resultados}

El programa de espectáculos analizado lleva por nombre "Ventaneando" el cual se presenta en un canal de televisión abierta. Este programa cuenta con una larga trayectoria en la televisión mexicana, su inicio data del año 1996 y en la actualidad se presenta en un horario de 3 a 4:30 p.m. Las emisiones analizadas son la consecución de una misma noticia: el abuso sexual realizado por un actor y cantante hacia su hija durante 9 años. En las emisiones se abordan las declaraciones emitidas por el agresor y personas cercanas a él, la denuncia interpuesta, así como los detalles del abuso sexual de parte de la hija, quien actualmente tiene 15 años.

\section{Programa de espectáculos \\ Estrategia discursiva 1. Minimización del abuso sexual}

Durante las emisiones transmitidas en el programa se realiza una minimización sistemática de los hechos que se relatan. Se establece en primera instancia que el acto cometido fue un abuso sexual durante años por el padre de la menor de edad; sin embargo, esto sucede con una recurrente duda de la violencia ejercida hacia la menor; conforme se avanza en la emisión se realiza una omisión de detalles e incluso se deja de mencionar la edad, parentesco y el tipo de violencia cometido, enfocándose únicamente en la carrera del agresor.

Aunado a ello, se sugiere al espectador a pensar que la denuncia establecida es resultado de otras motivaciones, por ejemplo:

$D B: Y$ todo pareciera indicar que considera que su mujer esta pues actuando en su contra de esta manera y...

PS: Como una venganza será

$D B$ : Como una venganza

PS: Pero el divorcio fue en 2017, fijate hace cuatro años

$P C$ : $Y$ no dijo nada entonces

$P S: Y$ entonces no pasó nada
Se utiliza a la madre de la menor como un medio para desviar la atención del agresor y se lleva a pensar que es ella quien, con otras motivaciones y gracias a sus recursos, mantiene en prisión a su ex marido posicionándolo como una víctima y olvidando la denuncia principal. Además, se argumenta a la temporalidad como una evidencia de que el acto por el que se le denuncia es poco creíble.

Sub-estrategia. Desvalorización de la denuncia establecida y de los procedimientos legales

Aun cuando en ocasiones se habla de que son las autoridades las encargadas de llevar el proceso correspondiente y solo puede hablarse de un culpable cuando se dé un veredicto, también es cierto que los conductores en diversos momentos expresan su inconformidad de que el agresor se encuentre en un proceso judicial, como se muestra a continuación:

PS: Hace unos pocos días cuando se anunció que Ricardo Crespo, este joven actor y cantante estaba en la cárcel porque había sido acusado de abusar sexualmente de su hija, yo, me dieron muchas dudas, porque dije cómo es posible que un hombre este en la cárcel si no se le ha probado.

El párrafo anterior muestra el poco conocimiento por parte del presentador del proceso judicial ante abuso sexual, pero también muestra la postura ante esta situación; se encuentra del lado del hombre que ha sido acusado y plantea ante el público la supuesta inocencia al mencionar que no hay nada que se haya probado $y$, por tanto, dicha persona no debería estar en la cárcel.

Cuando se presenta un dialogo centrado en los hechos que acontecen, y se realizan hipótesis que desembocan en la afirmación de la culpabilidad de la persona se recurre a desestimaciones como sucede a continuación:

LF: Ahora, si la fiscalía encontró elementos y lo tiene y detuvo, pues también tiene un valor eso ¿no?

$M C$ : Si, porque lo vinculo a proceso

LF: Obvio, lo vinculo a proceso

DB: Pero recordemos que a Kalimba también lo habian encontrado y al final no paso más

PS: Bueno, y al que supuestamente había matado al novio de Sharid Cid

$P C$ : Exacto

Se habla del procedimiento legal como algo en lo que se está en desacuerdo, se ignora los procedimientos seguidos por la fiscalía en estos casos y buscan persuadir al televidente de que la persona no debería estar en 
prisión preventiva; además, se utiliza como recurso de desacreditación casos en donde la denuncia por algún tipo de violencia o delito no procedió o bien, era falsa y se hace una comparación con el caso del que se habla; esto se utiliza para mostrar que aun que exista una denuncia y un procedimiento esto podría terminar siendo incorrecto y se defiende al agresor.

\section{Estrategia discursiva 2. Búsqueda de persuasión del televidente}

De manera constante se aprecia que los presentadores se encuentran defendiendo o justificando al agresor, las notas que se presentan están dirigidas a crear una imagen de víctima e inocencia del victimario. Esto es apreciable en el contenido que se presenta, es decir, en el material que utilizan para complementar la nota; en un momento dado se presenta el comunicado de prensa que el agresor emite, una parte de este es "hasta este momento he optado por no hacer declaraciones al respecto, pues yo mismo me encontré sorprendido, paralizado y atónito ante de la gravedad de los actos delictuosos que se me achacan" que es una forma en la que se deslinda totalmente de aquello que se le imputa; supone que toda la gente que ha escuchado de esta noticia ha reaccionado de esta forma y por tanto, al afirmar que el se siente de la misma forma, que le indignan los hechos que se relatan, esto le hace inocente. Si bien, esto no forma parte del discurso de quienes presentan el programa, el dar tiempo al aire a este comunicado de prensa (junto a otros recursos) en donde se habla de manera positiva del acusado, muestra una postura marcada del programa.

En diversos momentos se ve a los presentadores expresar su incredulidad del abuso sexual y dar apoyo a el agresor; este apoyo consiste en invisibilizar detalles, menospreciar a la víctima, mostrar que creen en su inocencia y aseverar que se trata de una difamación y que esperan pueda salir pronto de la cárcel y por tanto de las acusaciones en su contra.

PS: Pues sí, de la calumnia algo queda. Sales muy tocado de la cárcel y luego sales

señalado

$P C$ : Mira con que salga

$D B$ : Con que salga, si

\section{Sub-estrategia discursiva. Justificación de las acciones del agresor}

Se muestra una renuencia a presentarlo como alguien culpable o como un abusador sexual; constantemente se utilizan palabras como "presunto culpable" o se habla del "posible delito" lo que demuestra que no lo conceptualizan como una persona capaz de realizar alguna clase de violencia.

Se recurre a la argumentación en la búsqueda que justificar las acciones del agresor, en donde, se plantea que aun cuando y sus acciones fueron indebidas no debe juzgárseles con frases como "La mayoría de los malos actos digamos, son producto de la ignorancia nada más" en donde se busca que el espectador considere que el agresor desconoce que el acto de violencia que lleva a cabo y por tanto, se le impugne de la responsabilidad que se le alude.

\section{Sub-estrategia discursiva. Focalización positiva en el agresor}

El programa se enfoca únicamente en la visión del agresor, sobre los hechos solo se revela que madre e hija realizaron la denuncia por abuso sexual y se presenta una serie de detalles revelados por la hija; sin embargo, todo el demás contenido gira a la defensa del victimario; en las diferentes emisiones se presentan complementos como comunicados de prensa, fotografías o clips de entrevistas que buscan obtener de la audiencia una imagen positiva del agresor en donde se busca que el televidente lo vea como un hombre trabajador, alegre, profesional y con buenas relaciones sociales. Un ejemplo de ello es como presenta uno de los conductores al agresor como un "joven actor y cantante" asegurando una connotación positiva de la persona a la que se refiere con el adjetivo usado, además de mencionar sus habilidades para causar una buena impresión al espectador.

\section{Estrategia 3. Invalidación de la denunciante}

Se muestra una incredulidad constante a el acontecer de los hechos denunciados, mostrado en las dudas expresadas constantes sobre la veracidad de lo que se muestra en la denuncia como se observa en el siguiente fragmento: "PS: Pues sí, o sea, que, si la había toqueteado pero que la niña tenía la culpa, cosa que no entiendo si así fue" "DB: bueno, pero eso fue lo que declaro ella, ¿no?"; se alude a una provocación que pudiera llevar a cabo la menor de edad que propiciara la violencia que se ejerció en ella y se utilizan adjetivos despectivos que buscan poner en tela de juicio su capacidad para realizar la denuncia o ser consciente de los hechos que denuncia.

LF: Yo entiendo que hace referencia al padre

$R$ : ¿Al papá? Yo también entiendo eso

PS: Si, pero tenía 15 años

$D B$ : En esto no menciona una relación sexual

$P S$ : No, ella no dice que haya una relación 
LF: Pues sí, pero si se fue a hacer una prueba de embarazo entonces es porque hubo coito

MC: Exactamente

DB: O porque el temía que hubiera tenido con alguien más

En el fragmento anterior se debate si existió o no la penetración como parte de la violencia que se denuncia; como se observada dos de los conductores se esfuerzan por negar que haya existido una relación sexual forzada porque no es mencionado de forma explícita, aun cuando esto se encuentra de manera tácita en las declaraciones. Por si no fuera poco, se alude al ejercicio de la sexualidad de la joven para invisibilizar las posibles consecuencias del abuso sexual y al abuso mismo.

\section{Estrategia 4. Desviación de la atención del televidente del tema principal}

Además de mostrarse una serie de fotografías o videos que solo forman parte de la carrera artística del actor, se hace comentarios que pueden confundir a los espectadores y pensar que la denunciante daba consentimiento para la violencia sexual que vivió; además, se muestran una serie de detalles innecesarios sobre el abuso sexual:

Cuando tenía 5 años solo tengo el recuerdo de que mi papá me quitaba toda mi ropa y comenzaba a tocar todo mi cuerpo cuando mi mama salía de la casa, comenzó a tocarme mediante juegos, me decía que jugáramos al spa que consistía en que yo me desnudara y también mi papá lo hacía y con cremas y aceites me acariciaba

Estos detalles, no contribuyen al enriquecimiento de la nota y vulneran la intimidad de la víctima, además de fomentar el morbo de los espectadores.

El noticiero analizado en esta investigación se presenta en televisión abierta y lleva por nombre "Hechos meridiano" presentado en un horario de 2:00 a 3:00 p.m. Se analizaron dos de sus emisiones, una con fecha del 03 de febrero de 2021 y otra del 27 de abril de 2021. En primera emisión se encuentran dos noticias de violencia de género en distintos momentos de la emisión; la primera noticia dura alrededor de 54 segundos y la segunda, encontrándose en la sección de espectáculos, 1:30 segundos. En la segunda emisión se presenta un bloque consecutivo de noticias sobre violencia de género; mayormente violencia física, sexual y feminicidio; se presentan un total de cuatro noticias en un espacio de 2:25 segundos.

\section{Noticias}

Estrategia 1. Limitación de actuar de las mujeres que sufren violencia de género/ Denuncia ante las autoridades como una única forma de romper el ciclo de violencia

En las noticias que se presentan se hace hincapié en si la violencia que se muestra fue o no denunciada ante las autoridades, dando el mensaje a los televidentes que la única manera de terminar con un ciclo de violencia es a través de un procedimiento legal. De la misma manera, se observa un trato diferente a las mujeres cuya violencia no fue denunciada.

Cuando se habla de una denuncia hacia la sociedad no se le da la misma importancia o se minimiza como puede observarse en el siguiente ejemplo:

Cada vez más mujeres las que utilizan las redes sociales o ciertos.... entrevistas o testimoniales para hablar de abusos y me parece que ese sigue siendo el punto donde debemos poner atención y hacer que nuestro entorno sea más saludable y también que sea de más confianza para que las mujeres se sientan con la tranquilidad de ir a denunciar... ante las autoridades

Se asume que la única manera en que la mujer puede adquirir cierta agencia es a través de las autoridades y se desvalorizan otras formas de denuncia.

\section{Estrategia discursiva 2. Invisibilización de la violencia hacia la mujer más allá de la violencia física}

Las noticias en donde se presenta violencia de género giran en torno a la violencia sexual o a la violencia física además de hablarse de feminicidios; de los casos presentados en las emisiones que fueron analizadas solo se mostró un caso de violencia simbólica hacia la mujer; pero en este se pudo apreciar que el conductor se mostraba renuente a afirmar que lo que se presentaba era un tipo de violencia hacia la mujer.

\section{Conclusiones}

Tras el análisis realizado del discurso de los diversos participantes, se concluye que existe una tendencia a la protección del abusador; como puede observarse los conductores se encuentran constantemente invisibilizando la violencia ejercida hacia la mujer y apoyando en todo momento al agresor. En el programa de espectáculos es claro que no existe empatía hacia la víctima, a quien constantemente se menosprecia y desacredita, es el agresor quien obtiene toda la atención por parte de los conductores, lo que también tiene que ver con el poder que la persona sustenta, quienes se muestran parciales y olvidan la objetividad como parte del trabajo periodístico. 
Debe hacerse hincapié en que la violencia que se muestra al espectador, se vierte principalmente en la violencia física y sexual lo que influye en la conceptualización y el reconocimiento de la violencia de género en los diversos ámbitos en lo que puede presentarse, limitándose el conocimiento sobre las otras formas en las que se ejerce violencia hacia la mujer. Por último, y no menos importante, se encuentra una tendencia a establecer la denuncia penal como la única toma de acción viable y socialmente reconocida para poner un alto al ciclo de violencia, lo que invalida las otras maneras de tomar agencia de la mujer; aunado a ello se observa que, aun cuando se establece una denuncia formal ante las autoridades correspondientes esto no exime a la mujer del escrutinio público, se desestima la acusación o el delito de violencia de género aludiendo a la difamación y a la corrupción del sistema o a un procedimiento incorrecto por parte del sistema judicial.

\section{Referencias}

Galarza, E., Cobo, R., y Esquembre, M. (2016). Medios y violencia simbólica contra las mujeres. Revista latina de comunicación social, 71, 818-832. Recuperado de http://www.revistalatinacs.org/071/paper/1122/RLCS-paper1122.pdf

Gámez, M. (2012). Sobre los modos de visibilización mediático-política de la violencia de género en España: Consideraciones críticas para su reformulación. Revistas de ciencias sociales, 7 (2), 185-213. Recuperado de https://rua.ua.es/dspace/bitstream/10045/25937/1/OBETS $07 \quad 02 \quad 02$. pdf

Gámez, M., y Núñez, S. (2013). Medios, ética y violencia de género: más allá de la victimización. Asparkia, 24, 145-160. Recuperado de http://www.e-

revistes.uji.es/index.php/asparkia/article/view/1167/1043

Giberti, E., y Fernández, A. [Comps]. (1989). La mujer y la violencia invisible. Buenos Aires, Argentina: Editorial Sudamericana.

Magnabosco, M. (2014). El construccionismo social como abordaje teórico para la comprensión del abuso sexual. Revista de psicología, 32 (2), 220-242. Recuperado de http://www.scielo.org.pe/pdf/psico/v32n2/a02v32n2.pdf

Naciones Unidas. (1993). Declaración sobre la Eliminación de la Violencia contra la Mujer.

Núñez, S., y Fernández, D. (2015). Construcción identitaria del sujeto víctima de violencia de género: fetichismo, estetización e identidad pública. Revista Teknokultura, 12 (2), 267-284. Recuperado de https://revistas.ucm.es/index.php/TEKN/article/view/49506/46324

Sisto, V. (2012). Análisis del discursos y psicología: A veinte años de la revolución discursiva. Revista de psicología, 21 (1), 185-208. Recuperado https://revistahistoriaindigena.uchile.cl/index.php/RDP/article/view/1 9994/21157

Velázquez, S. (2003). Violencias cotidianas, violencia de género: escuchar, comprender, ayudar. Buenos Aires, Argentina: Paidós.
Zurbano, B., y Liberia, I. (2014). Revisión teórico-conceptual de la violencia de género y de su representación en el discurso mediático. Una propuesta de resignificación. Zer, 19 (36), 121-143. Recuperado de https://idus.us.es/bitstream/handle/11441/23624/zer36-06zurbano.pdf? sequence $=1 \&$ is Allowed $=y 1-163$. 